ज्ञ FRANÇAISE

$\lessgtr \mathrm{DE}$

딜 PEAGOGIE

\section{Revue française de pédagogie}

Recherches en éducation

182 | 2013

Connaissances et politiques d'éducation : quelles interactions?

\title{
Un modèle sociocognitif des apprentissages scolaires : style motivationnel de l'enseignant, soutien perçu des élèves et processus motivationnels
}

A sociocognitive model of academic learning: the motivational style of teachers, its perception by their students, and motivational processes

Nadia Leroy, Pascal Bressoux, Philippe Sarrazin et David Trouilloud

\section{(2) OpenEdition}

Édition électronique

URL : http://journals.openedition.org/rfp/4008

DOI : $10.4000 /$ rfp.4008

ISSN : 2105-2913

\section{Éditeur}

ENS Éditions

\section{Édition imprimée}

Date de publication : 28 août 2013

Pagination : 71-92

ISSN : 0556-7807

\section{Référence électronique}

Nadia Leroy, Pascal Bressoux, Philippe Sarrazin et David Trouilloud, « Un modèle sociocognitif des apprentissages scolaires : style motivationnel de l'enseignant, soutien perçu des élèves et processus motivationnels », Revue française de pédagogie [En ligne], 182 | 2013, mis en ligne le 28 août 2016, consulté le 19 avril 2019. URL : http://journals.openedition.org/rfp/4008 ; DOI : 10.4000/rfp.4008 


\title{
Un modèle sociocognitif des apprentissages scolaires: style motivationnel de l'enseignant, soutien perçu des élèves et processus motivationnels
}

\author{
Nadia Leroy, Pascal Bressoux, Philippe Sarrazin \\ et David Trouilloud
}

Dans le cadre de cette étude, nous proposons un modèle sociocognitif des apprentissages scolaires, basé sur la théorie de l'autodétermination et la théorie sociocognitive de Bandura (1986), qui intègre le style motivationnel de l'enseignant, la perception que les élèves en ont, trois variables motivationnelles (la motivation autodéterminée, l'amotivation et les croyances d'auto-efficacité) et le niveau scolaire des élèves évalué par des tests standardisés d'acquisitions. Les données ont été recueillies en 2003 à l'occasion de l'Évaluation-bilan-école conduite par la Direction de l'évaluation, de la prospective et de la performance du ministère de l'Éducation nationale. L'échantillon est constitué de 4402 élèves de CM2 et de leurs 326 enseignants. Des modèles multiniveaux conduits de manière séquentielle fournissent des résultats qui sont cohérents avec nos hypothèses théoriques. Dans ce qu'ils ont d'essentiel, les résultats montrent que le style motivationnel autorapporté par les enseignants est relié aux acquisitions scolaires des élèves et que cette relation transite par la perception que les élèves ont du style motivationnel autorapporté par leur enseignant, qui est reliée aux variables motivationnelles des élèves, elles-mêmes corrélées au niveau scolaire des élèves.

Mots-clés (TESE) : motivation, processus d'apprentissage, relations interpersonnelles, climat de la classe.

\section{INTRODUCTION}

Les recherches en sociologie de l'éducation se sont largement intéressées à la question des facteurs explicatifs des inégalités scolaires. Au-delà des caractéristiques propres aux élèves, certaines spécificités du contexte scolaire permettent d'expliquer une part significative de la variance des apprentissages (pour revue, Bressoux, 1994). Parmi celles-ci, les facteurs relatifs aux pratiques enseignantes s'avèrent particulièrement influents (Good \& Brophy, 2000). L'intérêt pour les mécanismes par lesquels l'enseignement produit ses effets sur les apprentissages apparaît dans les années 1970 (voir par exemple Anderson, 1970). En se focalisant sur les « processus humains implicites qui s'interposent entre les stimuli pédagogiques et les résultats de l'apprentissage » (Levie \& Dickie, 1973 ; cités par Doyle, 1986, p. 445), ces travaux ont permis de prendre davantage en compte les dimensions psychosociales et cognitives considérées comme constitutives de l'acte d'apprendre (Shuell, 1996).

Dans la lignée de ces études, l'objectif de cette recherche sera de mieux comprendre les mécanismes par lesquels le style motivationnel de l'enseignant affecte les apprentissages des élèves en considérant les processus motivationnels qui médiatisent cette influence. Le modèle élaboré et testé dans cette étude 
se fonde sur les apports issus de la théorie de l'autodétermination (TAD ; Deci \& Ryan, 2000, 2002 ; voir Sarrazin, Pelletier, Deci et al., 2011, pour un aperçu en français) et ceux de la théorie sociocognitive de Bandura (1986).

\section{La motivation : un processus médiateur?}

Dépendante des conditions d'enseignement et identifiée comme un déterminant des progrès des élèves (Ryan \& Patrick, 2001), la motivation est théoriquement considérée comme un processus médiateur de la relation enseignement-apprentissage. Si elle est fréquemment invoquée pour rendre compte de la réussite scolaire, on constate toutefois qu'elle est rarement étudiée en regard des liens qu'elle entretiendrait simultanément avec les contextes d'enseignement et avec les apprentissages. Parce qu'elle s'intéresse à la fois aux antécédents contextuels et aux conséquences de différentes formes de motivation, la TAD constitue un cadre théorique privilégié, qui a été utilisé ces dernières années dans différents domaines, dont celui de l'école, avec un bon pouvoir heuristique (voir Sarrazin, Pelletier, Deci et al., 2011, pour une revue).

\section{LA THÉORIE DE L'AUTODÉTERMINATION}

\section{Un continuum de motivations plus ou moins autodéterminées}

La TAD présume l'existence de différentes formes de motivation qu'il est possible d'ordonner sur un continuum d'autodétermination, c'est-à-dire en fonction du degré avec lequel le comportement est spontané, autonome et émane d'une force intérieure versus est obligé, contraint et émane d'une force extérieure. De la motivation la moins vers la plus autodéterminée, on trouve les régulations externe, introjectée, identifiée, intégrée et la motivation intrinsèque. La régulation externe renvoie aux comportements de l'individu régulés par des contingences externes auxquelles il ne s'identifie pas (par exemple obtenir une récompense, éviter une punition). La régulation introjectée correspond à l'engagement de l'individu dans l'activité afin d'éviter les conflits internes (par exemple culpabilité, honte). La régulation identifiée renvoie à un engagement de l'individu pour des raisons partiellement intériorisées (la tâche à accomplir revêt une importance personnelle). La régulation intégrée renvoie aux comportements réalisés parce qu'ils sont en complète harmonie avec l'identité et les valeurs centrales de l'individu. Enfin, la motivation intrinsèque - qui constitue l'archétype de la motivation autodéterminée - correspond à la pratique spontanée d'une activité pour l'intérêt qu'elle présente en elle-même. Les régulations externe et introjectée renvoient à des formes " contrôlées " de motivation (l'engagement de l'individu relève principalement de demandes sociales qui ne sont pas intériorisées), alors que les régulations identifiée, intégrée et intrinsèque représentent des formes « autonomes » de motivations (l'engagement de l'individu est spontané, volontaire, sans sentiment de contrainte) (Deci \& Ryan, 2000).

En plus de ces différentes régulations motivationnelles, la TAD propose également qu'une personne puisse faire preuve d'une absence relative de motivation, appelée amotivation. Ce concept n'est pas considéré comme un élément appartenant au continuum mais doit néanmoins être pris en compte puisqu'il permet lui aussi d'expliquer un certain nombre de comportements manifestés par les élèves. L'amotivation serait ainsi à l'œuvre lorsque l'individu ne parvient pas à faire le lien entre ses comportements et les résultats qu'il obtient. Cet état est proche de ce que Seligman (1975) appelle la « résignation apprise ».

\section{Les conséquences associées aux différentes régulations motivationnelles}

Les travaux fondés sur le continuum d'autodétermination indiquent que les différents types de régulations mènent à des conséquences qui sont d'autant plus adaptatives que la motivation est autonome (pour revue, voir Deci \& Ryan, 2002). Les études réalisées dans le domaine éducatif montrent que les formes autonomes de la motivation sont reliées à un meilleur apprentissage conceptuel, une plus grande réussite scolaire, des émotions positives, une plus grande satisfaction à l'égard de l'école, une meilleure estime de soi et des perceptions de compétence plus élevées (pour revue, voir Niemiec \& Ryan, 2009 ; Reeve, 2002). Les formes contrôlées de motivation sont associées à des conséquences négatives, notamment en termes de poursuite d'études (Vallerand \& Bissonnette, 1992). Enfin, l'amotivation, qui est associée négativement au bien-être psychologique, produit les conséquences les plus négatives comme une chute de la persévérance, l'abandon et in fine la baisse des performances (voir Niemiec \& Ryan, 2009 ; Reeve, 2002, pour une revue).

Au vu des bénéfices académiques associés aux formes de motivations les plus autonomes, promouvoir ce type de régulations chez les élèves constitue un enjeu majeur. Parmi les facteurs sociaux susceptibles de nourrir la motivation autodéterminée des élèves, 
la TAD souligne le rôle crucial du style motivationnel adopté par l'enseignant (Tessier, Sarrazin \& Trouilloud, 2006).

\section{L'effet des facteurs sociaux sur la motivation : le rôle du style motivationnel}

Selon la TAD, l'environnement social affecterait la motivation de l'individu via son action sur la satisfaction de trois besoins psychologiques fondamentaux : les besoins de compétence, d'autonomie et de proximité sociale (Deci \& Ryan, 2000). La catégorisation des environnements sociaux susceptibles de favoriser ou d'entraver la motivation s'organise donc autour de ces trois besoins. L'autonomie fait référence au degré avec lequel l'individu se sent " à l'origine " de ses actions plutôt qu'un « pion » qu'on manipule (De Charms, 1968) et fait l'expérience d'un choix qui est concordant avec ses intérêts et valeurs en l'absence de tout sentiment de pression (Deci, 1975). La compétence relève quant à elle du sentiment d'utiliser ses capacités de manière efficace (White, 1959). Enfin, la proximité sociale renvoie au besoin de se sentir connecté affectivement ou d'appartenir à un groupe (Baumeister \& Leary, 1995). Lorsque ces trois besoins sont satisfaits par l'environnement, l'individu s'identifie progressivement aux demandes sociales (par exemple les tâches proposées par l'enseignant) et les intègre à son propre registre de valeurs pour les faire siennes. Ainsi, plus l'individu percevra dans son environnement social des sources de satisfaction de ses besoins psychologiques, plus sa motivation sera autodéterminée. La perception d'un soutien des besoins est à concevoir comme une variable située à l'interface entre l'environnement social et la motivation de l'individu.

Le style motivationnel adopté par un enseignant fait partie des variables environnementales susceptibles de satisfaire les besoins des élèves (Reeve, 2002 ; Tessier, Sarrazin \& Trouilloud, 2006). Des chercheurs tels que Reeve $(2002,2009)$ ont proposé de classifier les styles motivationnels le long d'un continuum ayant pour extrémités, d'un côté le "style soutenant l'autonomie » et de l'autre le " style contrôlant ». Le style soutenant l'autonomie renvoie aux comportements qu'une personne adopte envers une autre personne en vue de promouvoir les intentions volitives ainsi que les besoins psychologiques de cette dernière. Un enseignant soutenant l'autonomie cherchera à identifier les ressources internes de ses élèves en créant des conditions favorables à la satisfaction de leurs besoins motivationnels de façon à encourager le processus d'internalisation et leur motivation intrinsèque. Au niveau des pratiques enseignantes, ce style motivationnel se manifeste par des opportunités laissées aux élèves de décider, de s'exprimer et de faire des choix (soutien du besoin d'autonomie), par des indications données au sujet des progrès réalisés ou encore des encouragements aux efforts (soutien du besoin de compétence), une grande écoute, des conduites empathiques et l'acceptation des difficultés que peuvent éprouver les élèves (soutien du besoin de proximité sociale) (pour un développement plus approfondi, voir Sarrazin, Pelletier, Deci et al., 2011 ; Tessier, Sarrazin \& Trouilloud, 2006). De nombreuses études ont montré que les élèves dont les enseignants soutiennent l'autonomie manifestent une plus grande motivation autodéterminée (Chirkov \& Ryan, 2001), ont de meilleurs résultats scolaires (Black \& Deci, 2000) et affichent un sentiment de compétence plus élevé (Ryan \& Grolnick, 1986).

Par opposition, le style contrôlant est défini comme un ensemble de pratiques accentuant le poids des pressions et ayant pour but d'inciter les élèves à agir d'une façon spécifique (Deci \& Ryan, 1987). Les enseignants qui adoptent un tel style utilisent des renforcements, un langage plus directif et des modes de communication contraignants pour amener les élèves à faire ce qu'ils attendent (Reeve, 2002, 2009 ; Reeve \& Jang, 2006). Ce style motivationnel se manifeste par un monopole de la parole, moins de temps accordé à la résolution d'exercices, une utilisation fréquente de récompenses/punitions contingentes, de feedback concernant l'intelligence ou bien encore plus de critiques et de désapprobation à l'égard des élèves (Deci, Connell \& Ryan, 1989). De tels comportements sont défavorables à la satisfaction des besoins motivationnels des élèves et favorisent in fine les formes plus contrôlées de la motivation (Deci, Koestner \& Ryan, 1999) et l'amotivation (Boggiano, 1998).

\section{L'effet des perceptions de soi sur la motivation : le rôle des croyances d'auto-efficacité}

Si le degré d'autodétermination de la motivation est en partie dépendant des facteurs sociaux et plus particulièrement de la capacité du style motivationnel de l'enseignant à favoriser les besoins psychologiques des élèves, d'autres travaux font également apparaître que les perceptions que les élèves développent à propos d'eux-mêmes sont des facteurs qui permettent de mieux comprendre les motifs qui sous-tendent leur motivation. À ce titre, les travaux conduits par Bandura (1997) et par Schunk (Schunk, 1991 ; Pintrich \& Schunk, 1996) permettent d'envisager les jugements cognitifs formulés par l'individu quant à sa capacité à atteindre un objectif 
ou à accomplir une tâche donnée comme de puissantes ressources motivationnelles. Ces perceptions, appelées croyances d'auto-efficacité (Bandura, 1986), déterminent notamment la qualité de la motivation ainsi que le niveau d'engagement, de persévérance de l'individu face à la difficulté. Bien que distinctes au plan terminologique, croyances d'auto-efficacité et compétence perçue partagent certaines proximités tant au plan conceptuel (Bandura, 1997 ; Pajarès, 1996 ; Ryan \& Deci, 2000) qu'empirique (Pietsch, Walker \& Chapman, 2003). Les croyances d'auto-efficacité étant plus sensibles aux variations de l'environnement immédiat de la classe que la compétence perçue (Skaalvik \& Hagtvet, 1990 ; Wigfield, Eccles, Yoon et al., 1997), elles se révèlent pertinentes à intégrer dans les études consacrées à l'effet de l'environnement scolaire. Elles constituent un facteur particulièrement intéressant à considérer pour rendre compte des apprentissages dans la mesure où elles affectent la réussite scolaire directement mais également indirectement via le niveau de motivation des élèves (Goetz, Cronjaeger, Frenzel et al., 2010). Comme dans les travaux basés sur la TAD, ceux qui s'inscrivent dans le cadre du modèle de Bandura (voir Bandura, 1997) ont souligné l'impact de l'environnement social, et en particulier celui des "persuasions verbales", sur les croyances d'auto-efficacité. Plus l'individu se sent encouragé et soutenu dans la réalisation d'une tâche particulière, plus ses croyances d'auto-efficacité sont élevées. Dans le contexte scolaire, les messages adressés par l'enseignant à ses élèves constituent une importante source de persuasions verbales. Par leurs encouragements et feed-back sur les progrès réalisés, les enseignants peuvent influencer les croyances d'auto-efficacité des élèves.

\section{VERS UN MODĖLE MOTIVATIONNEL DES APPRENTISSAGES SCOLAIRES}

Les travaux menés dans le champ de l'éducation et ceux conduits dans le cadre de la psychologie apportent donc des éclairages qui se complètent dans la mesure où les premiers ont permis de mettre en évidence les pratiques éducatives et le type de relations interpersonnelles qui influencent les croyances et les attitudes des élèves alors que les seconds éclairent davantage sur la manière dont ces processus sont liés à l'engagement des élèves à l'école. Toutefois, si la littérature fait apparaître la motivation non seulement comme une variable dépendante des conditions d'enseignement mais également comme une variable indépendante affectant la réussite scolaire, ces deux voies de recherche trahissent un manque d'intégration théorique. De fait, bien que la TAD propose que les facteurs sociaux affectent les processus motivationnels qui, à leur tour, affectent les apprentissages, les études en contexte scolaire ayant cherché à tester, au sein d'un modèle intégrateur, le statut des processus motivationnels demeurent à l'heure actuelle encore rares (pour revue, voir Niemiec \& Ryan, 2009).

Par ailleurs, lorsque l'on s'intéresse plus précisément aux études traitant de l'effet des facteurs sociaux sur la motivation des élèves, on peut souligner l'existence de deux approches distinctes qui ne sont pas sans poser problème au plan théorique. D'un côté, des études se sont intéressées à l'effet direct du contexte social sur la motivation (par exemple Grolnick \& Slowiaczek, 1994) sans considérer l'interprétation faite par l'individu des signaux contextuels. D'autres travaux ont porté exclusivement sur les liens entre la perception par l'individu de l'environnement social et sa motivation (par exemple Kaplan \& Maehr, 1999), supposant quant à eux une homologie entre l'environnement social objectif et la perception que l'individu en a. Une analyse des effets du style motivationnel de l'enseignant qui se veut en adéquation avec les approches sociocognitives (comme la TAD ou la théorie sociocognitive de Bandura) nécessiterait de croiser ces deux perspectives. S'il semble important d'avoir une idée aussi objective que possible des comportements manifestés par l'enseignant, la théorie de l'évaluation cognitive - l'une des mini-théories intégrée dans la TAD (voir Deci \& Ryan, 2000) - tout comme la théorie sociocognitive de Bandura (1986) insistent sur le fait que ce ne sont pas les comportements du superviseur en tant que tel qui influencent la motivation du supervisé, mais plutôt la perception que ce dernier en a. À l'exception de rares études (Reeve \& Jang, 2006 ; Skinner \& Belmont, 1993) qui intègrent au sein d'une même chaîne causale le style de l'enseignant et la perception que les élèves en ont, la plupart des travaux se focalisent sur une source d'information unique laissant inconnus les liens entre le style de l'enseignant autorapporté par ce dernier et les perceptions qu'en ont les élèves.

\section{LA PRÉSENTE ÉTUDE}

\section{Objectif}

L'objectif de la présente étude est de proposer un modèle explicatif des apprentissages intégrant à la fois les facteurs contextuels (c'est-à-dire le style 


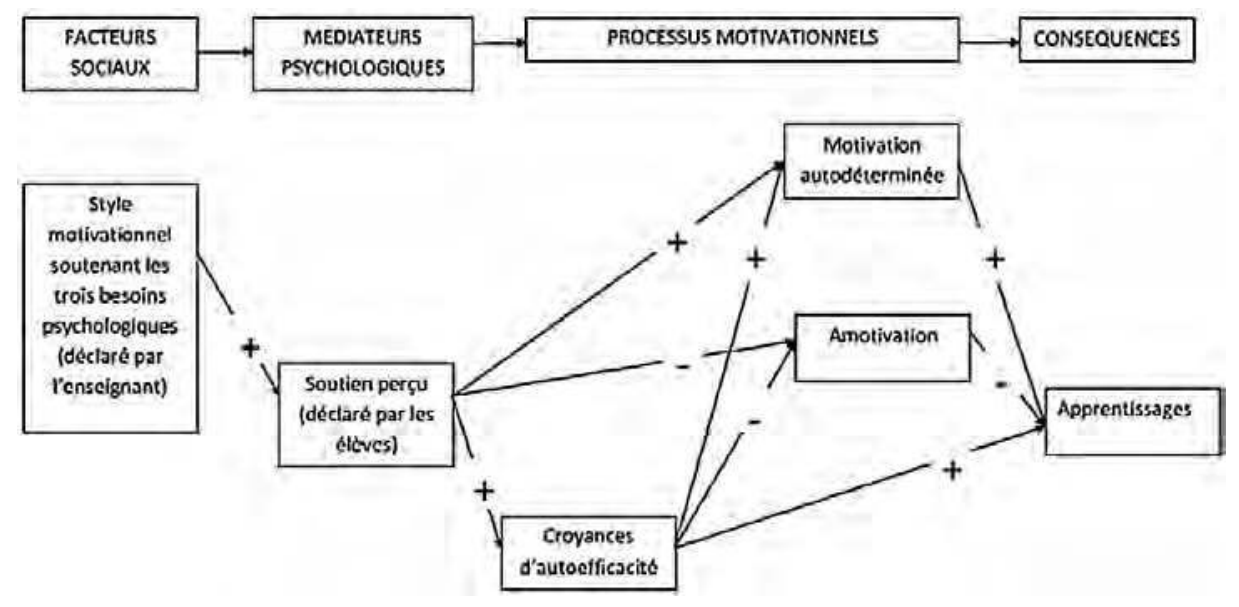

motivationnel de l'enseignant) et les processus motivationnels, et qui considère l'environnement social à la fois du point de vue de l'élève et de l'enseignant. Notre modèle théorique (voir figure 1) repose sur la séquence causale présumée par la TAD (Deci \& Ryan, 2002). Le facteur social considéré ici correspond au style motivationnel autorapporté par l'enseignant. Les variables motivationnelles étudiées sont le degré d'autodétermination des motivations, l'amotivation, ainsi que les croyances d'auto-efficacité. La perception par les élèves du style motivationnel de leur enseignant s'interpose entre le style motivationnel autorapporté par l'enseignant et les processus motivationnels des élèves. En cela, elle occupe le statut de médiateur psychologique dans la séquence causale. Enfin, les conséquences associées à ces processus motivationnels qui seront étudiées ici sont les apprentissages scolaires objectivés par des tests standardisés d'acquisitions. Nous testerons ainsi les relations entre quatre niveaux de la séquence causale : le style motivationnel autorapporté par l'enseignant, la perception que les élèves en ont, les variables motivationnelles des élèves et, enfin, leurs apprentissages scolaires.

\section{Hypothèses}

Concernant les liens entre les processus motivationnels des élèves et leurs apprentissages, nous formulons les hypothèses suivantes:
- les degrés d'autodétermination et d'amotivation des élèves sont reliés respectivement positivement et négativement aux apprentissages des élèves ;

- les croyances d'auto-efficacité des élèves sont reliées positivement aux apprentissages de manière à la fois directe et indirecte via la médiation de la motivation autodéterminée et de l'amotivation.

Concernant les liens entre le style motivationnel de l'enseignant perçu par les élèves et les apprentissages de ces derniers :

- la perception par les élèves d'un style motivationnel qui soutient leur autonomie est reliée positivement, mais de manière indirecte, à leurs apprentissages ; ce lien est médiatisé positivement par la motivation autodéterminée et les croyances d'auto-efficacité, et négativement par l'amotivation.

Enfin, concernant les liens entre le style motivationnel autorapporté par l'enseignant et les processus motivationnels des élèves :

- un style d'enseignement qui soutient l'autonomie des élèves est relié positivement d'une part aux croyances d'auto-efficacité et à la motivation autodéterminée des élèves et négativement d'autre part à l'amotivation ; ces liens sont médiatisés par la perception des élèves relative au style d'enseignement de leur enseignant. Cette dernière est reliée, quant à elle, positivement aux croyances d'auto-efficacité et à la motivation autodéterminée, et négativement à l'amotivation. 


\section{MÉTHODE}

\section{Participants}

L'étude repose sur l'Évaluation-bilan-école (EBE) conduite en 2003 par la Direction de l'évaluation, de la prospective et de la performance (DEPP) du ministère de l'Éducation nationale. L'échantillon, représentatif de la population nationale correspondante, comprend 6109 élèves de CM2 qui appartiennent à 269 écoles et 336 classes. On dispose pour les élèves de renseignements sociodémographiques (sexe, profession des parents, année et mois de naissance) et de renseignements sur le déroulement de la scolarité (redoublement ou non, soutien ou non, enseignement pré-élémentaire). L'échantillon d'enseignants est constitué de 336 participants. On dispose pour eux également d'un ensemble de renseignements sociodémographiques (sexe, année de naissance) et professionnels (formation initiale et continue, ancienneté dans l'Éducation nationale, emploi à temps plein ou partiel).

\section{Instruments}

À la fin de l'année scolaire 2002-2003, les élèves ont passé une épreuve et renseigné, au cours de séances de passation collectives, un livret regroupant tous les questionnaires nécessaires à cette étude. Un questionnaire avait également été transmis aux enseignants au cours de cette même période. Tous les participants avaient été informés du caractère anonyme de l'étude. Pour chacun des items proposés, les répondants devaient indiquer à quel point ils étaient d'accord avec les phrases proposées sur une échelle de Likert en cinq points allant de (1) « pas du tout vrai » à (5) « tout à fait vrai ».

\section{Mesure de l'environnement social : le style motivationnel de l'enseignant}

Une version adaptée et traduite du Learning Climate Questionnaire (LCQ ; Williams \& Deci, 1996) a été utilisée pour évaluer le style motivationnel de l'enseignant. Cette échelle est composée de quatorze items auxquels les enseignants ont eu à répondre en exprimant leurs " choix en matière d'enseignement ». Ce questionnaire mesure la propension du style motivationnel de l'enseignant à soutenir l'autonomie (par exemple « En classe, je laisse à mes élèves des possibilités de choisir certaines choses... "), à encourager les sentiments de compétence (par exemple « En classe, quand je m'adresse à un élève, je fais en sorte que ce que je lui dis soit de nature à renforcer sa confiance en lui dans la matière ") et de proximité sociale des élèves (par exemple «En classe, j'essaie de me mettre à la place de mes élèves, en particulier ceux qui ont des difficultés "). Une analyse en composantes principales (ACP) a révélé que tous les items (sauf deux, retirés des analyses ultérieures) sont corrélés sur le premier facteur qui explique $30,1 \%$ de la variance totale avec une valeur propre égale à 4,21. Dans la mesure où il n'y a pas d'autres facteurs spécifiques clairement interprétables, nous avons constitué un score global de "soutien des besoins motivationnels " en faisant la moyenne des scores obtenus à chacun des items $(\alpha=0,82)$.

\section{Le médiateur psychologique : le style motivationnel de l'enseignant perçu par les élèves}

Le même questionnaire que celui utilisé pour les enseignants a été complété par les élèves afin de mesurer leur perception du style motivationnel de leur enseignant. Les élèves devaient se prononcer sur la manière dont ils pensaient que leur enseignant soutenait leurs besoins d'autonomie (par exemple « Dans la classe, mon enseignant-e me laisse des possibilités de choisir certaines choses, comme les textes à travailler, les livres à lire, quand faire telle ou telle chose, etc. "), de compétence (par exemple "Ce que me dit mon enseignant-e augmente la confiance que j'ai en moi en classe ") et de proximité sociale (par exemple « Je sens que mon enseignant-e m'accepte comme je suis"). Comme pour les enseignants, une ACP a montré un premier facteur (valeur propre $=5,41$; variance expliquée $=38,65 \%$ ) auquel tous les items corrèlent fortement (sauf un, retiré des analyses ultérieures), ce qui tend à indiquer l'existence d'une dimension principale du climat perçu. Nous avons là aussi constitué un score global de perception par les élèves d'un style d'enseignement qui soutient leurs besoins psychologiques. La consistance interne de ce dernier est très satisfaisante $(\alpha=0,88)$.

Les variables motivationnelles: la motivation autodéterminée, l'amotivation et les croyances d'auto-efficacité des élèves

\section{La motivation}

L'évaluation de la motivation des élèves a été effectuée grâce à l'Academic Self-Regulation Questionnaire (SRQ-A) de Ryan et Connell (1989). Les 36 items utilisés visaient à mesurer les régulations externe (9 items ; par exemple « J'essaie de bien faire à l'école, car on m'a promis des récompenses si je me débrouille bien "; $\alpha=0,72$ ), introjectée (9 items ; par exemple " J'essaie de bien faire à l'école parce que je veux que mon professeur pense que je suis un bon élève "; $\alpha=0,84$ ), identifiée (7 items ; par exemple « J'essaie 
de bien faire à l'école parce que je considère que c'est important »; $\alpha=0,80$ ), intrinsèque (7 items ; par exemple « Je fais mon travail scolaire parce que j'aime bien ça "; $\alpha=0,82$ ), ainsi que l'amotivation (4 items ; par exemple " Franchement, je n'arrive pas à voir à quoi ça sert de travailler à l'école "; $\alpha=0,76$ ).

Conformément aux travaux antérieurs (Ryan \& Connell, 1989), un indice d'autodétermination a été calculé en pondérant les scores obtenus à chacune des régulations par un coefficient qui correspond à la place de celle-ci sur le continuum d'autodétermination, suivant la formule suivante :

Indice d'autodétermination $=(2 \times$ intrinsèque $)+$ identi-

fiée - introjectée - $(2 \times$ externe)

Compte tenu des échelles adoptées, l'indice peut théoriquement varier de +12 à -12 . Plus ce score est élevé, plus le degré d'autodétermination de la motivation est fort (moyenne $=1,08$; écart-type $=2,88$ ) .

\section{Les croyances d'auto-efficacité}

L'échelle visant à mesurer les croyances d'autoefficacité a été élaborée pour les besoins de l'étude et s'inspire des items proposés dans le Motivated Strategies for Learning Questionnaire de Pintrich et De Groot (1990) ainsi que dans le Perceived Competence Scale (Williams, Freedman \& Deci, 1998 ; Williams \& Deci, 1996). Les élèves devaient se prononcer sur leurs croyances d'auto-efficacité (par exemple « Je me sens largement capable d'apprendre ce que mon enseignant-e nous enseigne »). Les résultats montrent que les cinq items de l'échelle ont une bonne consistance interne $(\alpha=0,79)$.

\section{Les conséquences : les acquisitions scolaires des élèves}

Les épreuves standardisées de fin d'année ont été élaborées par la DEPP. Elles portaient principalement sur la lecture, mais une partie des épreuves portait également sur les mathématiques, les sciences, la géographie et l'histoire. Elles étaient constituées d'exercices écrits et oraux (uniquement pour la lecture dans ce dernier cas). Nous avons utilisé le score global d'acquisitions (construit par la DEPP sur la base de modèles de réponse à l'item) qui concernait 229 items. Ce score, qui indique un niveau général d'acquisitions scolaires, démontrait une excellente cohérence interne $(\alpha=0,97)$ et variait de 71,55 à 431,27 points (avec une moyenne de 251,5 et un écart-type de 49,5 ).

\section{Données manquantes}

Des données manquantes ont quelque peu réduit la taille de l'échantillon initial EBE construit par la DEPP. La réalisation de plusieurs séries d'imputations n'a pas permis de récupérer toutes les données manquantes pour l'analyse. Retenons donc que l'échantillon sur lequel nous réalisons les analyses ne peut être considéré comme strictement représentatif de la population nationale correspondante. Nous pouvons néanmoins penser, sous l'hypothèse que les données manquantes ont une distribution proche d'une distribution aléatoire, qu'il en a des caractéristiques relativement proches. Les analyses porteront sur un échantillon de 4402 élèves et 326 enseignants.

\section{Analyses et description de la démarche de modélisation}

Cette étude impliquant des unités statistiques situées à différents niveaux hiérarchiques (c'est-à-dire des élèves dans des classes différentes), nous avons traité les données via la modélisation multi-niveaux (MMN ; voir Bressoux, 2010). Pour cela, différents modèles ont été successivement testés. Afin de rendre compte de l'enchaînement des variables et de tester leurs effets directs et indirects, l'ordre d'introduction des variables a été effectué de la variable la plus proximale à la plus distale aux acquisitions scolaires.

\section{RÉSULTATS}

Pour le modèle de base des apprentissages, nous posons que les acquisitions ainsi que les processus motivationnels des élèves dépendent de certaines de leurs caractéristiques individuelles : caractéristiques sociodémographiques et caractéristiques scolaires. Les variables retenues sont celles qui apparaissaient significatives dans au moins un des modèles séquentiels spécifiés, à savoir : la profession du père, la taille de la fratrie, la période de naissance (début, milieu, fin d'année), le déroulement de la scolarité (en avance, " à l'heure ", en retard) et le fait que l'élève soit ou non en situation de redoublement au cours de son année de CM2. Ces variables ayant une origine antérieure à la période d'étude, elles seront considérées comme des variables de contrôle. Elles sont toutefois nécessaires à nos analyses pour dégager des effets nets des variables qui nous intéressent. 
Tableau 1. Moyennes, écarts-type et matrice de corrélations

\begin{tabular}{|l|c|c|c|c|c|c|c|c|}
\hline Variables & $\mathbf{M}$ & $\mathbf{E T}$ & $\mathbf{M i n}-\mathbf{M a x}$ & $\mathbf{2}$ & $\mathbf{3}$ & $\mathbf{4}$ & $\mathbf{5}$ & $\mathbf{6}$ \\
\hline $\begin{array}{l}\text { Style motivationnel } \\
\text { autorapporté par } \\
\text { l'enseignant }\end{array}$ & 4,32 & 0,38 & $1,36-5,00$ & $0,12^{* * *}$ & $0,05^{* * *}$ & $-0,07^{* * *}$ & $0,03^{*}$ & $0,04^{*}$ \\
\hline $\begin{array}{l}\text { Style de l'enseignant perçu } \\
\text { par les élèves }\end{array}$ & 3,26 & 0,83 & $1,00-5,00$ & - & $0,18^{* * *}$ & $-0,16^{* * *}$ & $0,21^{* * *}$ & $-0,06^{* * *}$ \\
\hline Indice d'autodétermination & 1,12 & 2,88 & $-9,54-11,67$ & & - & $-0,46^{* * *}$ & $0,28^{* * *}$ & $0,19^{* * *}$ \\
\hline Amotivation & 1,59 & 0,86 & $1,00-5,00$ & & & - & $-0,17$ & $-0,22^{* * *}$ \\
\hline Croyances d'auto-efficacité & 3,61 & 0,76 & $1,00-5,00$ & & & & - & $0,33^{* * *}$ \\
\hline Score d'acquisitions & 255,91 & 48,54 & $97,63-431,26$ & & & & & - \\
\hline
\end{tabular}

Note $: \mathrm{N}=4402 ; \mathrm{M}=$ moyenne $; \mathrm{ET}=$ écart-type $;$ Min-Max = minimum-maximum. ${ }^{*}: \mathrm{p}<0,05 ;{ }^{* *}: \mathrm{p}<0,01 ;{ }^{\star \star *}: \mathrm{p}<0,001$.

Les résultats ${ }^{1}$ sont présentés de façon séquentielle correspondant aux modélisations des différentes étapes du processus qui génèrent les acquisitions scolaires. Pour chacun des modèles présentés, les estimations reportées correspondent à celles produites par le logiciel SAS version 9.2 après suppression des variables non significatives. Ces dernières apparaissent toutefois dans les tableaux pour signifier que leurs effets ont bien été testés dans les analyses, mais, conformément à la nécessité de produire des modèles parcimonieux, les estimations reportées ont quant à elles été produites par des modèles excluant les variables non significatives. Les statistiques descriptives et les interrelations entre variables sont présentées dans le tableau 1.

\section{Expliquer les acquisitions scolaires à partir des processus motivationnels et du style motivationnel de l'enseignant}

Du modèle vide (modèle $A$, cf. tableau 2 en annexe), on relève que la part de variance inter-classe s'élève à $15,96 \%$ et que la part de variance intra-classe (ou inter-élèves) est de 84,04\%. Nous avons également testé un modèle dans lequel la variance pouvait être décomposée en un troisième niveau (niveau établissement) mais ce dernier n'ajustait pas mieux les données que le précédent. Notre analyse portera donc sur un modèle à deux niveaux.

Un modèle intermédiaire (modèle A bis, cf. tableau 2 en annexe) n'incluant que les caractéristiques sociodémographiques et scolaires des élèves a montré un meilleur ajustement que le modèle vide $\left(\Delta_{\mathrm{D}}=1131\right.$, pour $17 \mathrm{ddl}$ [degré de liberté]) tout en démontrant des parts de variances résiduelles significatives malgré une baisse de $42 \%$ de la variance au niveau des classes et de $21,5 \%$ de la variance au niveau des élèves. Ceci tend à indiquer que des différences subsistent à l'intérieur des classes entre les élèves (en intra) et entre les classes (en inter) malgré le contrôle de caractéristiques sociodémographiques et les éventuels effets de composition. II s'agit là de notre modèle de base, celui que nous allons maintenant compléter en ajoutant les variables relatives aux processus motivationnels (modèle A').

L'introduction des processus motivationnels (c'est-àdire indice d'autodétermination, niveau d'amotivation et croyances d'auto-efficacité) et des perceptions des élèves relatives au style motivationnel de leur enseignant a permis de faire ressortir plusieurs variables significativement reliées aux acquisitions scolaires (modèle A', cf. tableau 2 en annexe).

Des analyses préliminaires ayant laissé penser que l'indice d'autodétermination pouvait entretenir une relation non linéaire avec les scores d'acquisitions, nous avons spécifié une fonction quadratique. Cette dernière s'est révélée significative, indiquant que le lien entre le niveau d'autodétermination de la motivation et les résultats scolaires est quasi nul pour les valeurs faibles et moyennes de l'indice (c'est-à-dire pour une motivation autodéterminée faible et modérée), puis s'accroît de plus en plus au fur et à mesure que la motivation autodéterminée augmente (voir figure 2). 
Figure 2. Relation entre l'indice d'autodétermination et le score d'acquisitions des élèves

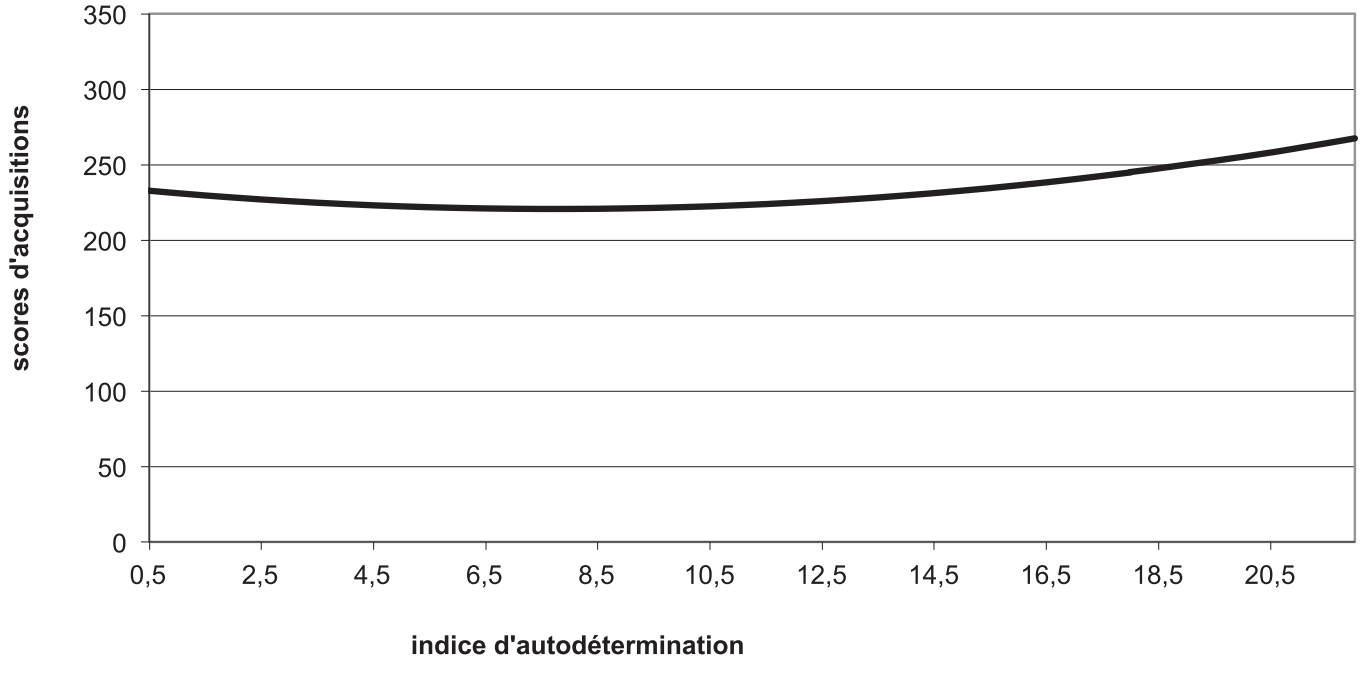

L'amotivation est quant à elle reliée négativement aux acquisitions scolaires $(\beta=-0,12)$ : les élèves amotivés vis-à-vis de l'école ont un niveau d'acquisitions moins élevé. En outre, les croyances d'auto-efficacité expliquent une part significative de la variance résiduelle des acquisitions, après avoir contrôlé l'effet des variables motivationnelles. Conformément à notre hypothèse, les croyances d'auto-efficacité sont positivement reliées aux acquisitions $(\beta=0,24)$, l'élévation d'un point d'écart-type dans le score d'auto-efficacité occasionne un gain de 0,24 point d'écart-type au plan des acquisitions de fin d'année.

Contrairement à nos attentes, le style motivationnel de l'enseignant perçu par les élèves est relié négativement aux acquisitions scolaires $(\beta=-0,13)$ : plus les élèves perçoivent que leur enseignant soutient leurs besoins fondamentaux et moins leurs acquisitions scolaires sont élevées. Nous avons testé un modèle avec fonction quadratique de manière à savoir s'il n'existait pas une valeur optimale de soutien perçu à partir de laquelle cette relation négative pouvait s'inverser, mais les résultats nous ont indiqué un coefficient non significatif. Nous reviendrons en détail sur ce résultat dans la discussion. Le style motivationnel autorapporté par l'enseignant n'est en revanche pas relié avec les acquisitions scolaires, le coefficient étant non significatif.

L'ajout des variables relatives aux processus motivationnels dans cette modélisation des acquisitions a occasionné une baisse de $13,27 \%$ de la variance intra comparativement à notre modèle de base, laissant toutefois les parts de variances résiduelles aux deux niveaux très significatives.

\section{Expliquer l'indice d'autodétermination à partir du style motivationnel de l'enseignant}

Dans un premier temps, nous avons introduit dans la modélisation de l'indice d'autodétermination le style motivationnel autorapporté par l'enseignant. Cette variable, lorsqu'elle est introduite seule, est significativement reliée à la motivation $(\beta=0,13)$. Toutefois, dès que la perception des élèves relative au style motivationnel de leur enseignant est introduite dans le modèle (modèle B', cf. tableau 3 en annexe), seule cette dernière variable est positivement reliée à la motivation autodéterminée $(\beta=0,13)$. Ce résultat suggère que les perceptions qu'ont les élèves du style de leur enseignant médiatisent l'effet de ce dernier sur leur motivation. Ce résultat est corroboré par le gain de variance expliquée ainsi que la décroissance de la déviance qui se révèlent plus élevés dans le modèle introduisant la perception des élèves que dans celui où le style motivationnel est le seul prédicteur. Dans le premier cas, on a un gain de $18,9 \%$ de la variance de niveau inter et $2,62 \%$ au niveau intra avec une décroissance de la déviance égale à 141,9 alors que dans le second cas, le gain est nul en intra et s'élève à $3,77 \%$ en inter avec une décroissance de la déviance égale à 5,3 .

Par ailleurs, les croyances d'auto-efficacité de l'élève sont reliées significativement et positivement à l'indice d'autodétermination $(\beta=0,24)$ : plus les 
élèves se croient capables de réaliser les tâches qui leur sont proposées à l'école, plus leur motivation est autodéterminée.

\section{Expliquer l'amotivation à partir du style motivationnel de l'enseignant}

Conformément à nos hypothèses, les résultats issus de la modélisation de l'amotivation (modèle C', cf. tableau 4 en annexe) indiquent que cette variable est reliée négativement au style motivationnel autorapporté de l'enseignant, à la perception qu'en ont les élèves, ainsi qu'aux croyances d'auto-efficacité. L'introduction du style motivationnel autorapporté par les enseignants dans le modèle occasionne une réduction de la variance résiduelle de l'amotivation d'environ $8 \%$ au niveau inter alors que l'introduction de la perception par les élèves du style motivationnel de leur enseignant occasionne quant à lui une réduction de variance résiduelle de $16,7 \%$ en inter et de $1,5 \%$ en intra. Le fait que l'introduction de la perception des élèves n'entraîne pas de disparition de l'effet du style motivationnel autorapporté par l'enseignant sur l'amotivation, mais simplement une légère réduction, suggère que la relation négative entre l'amotivation et le style motivationnel autorapporté par les enseignants n'est que partiellement médiatisée par la perception que les élèves en ont. Ainsi, plus les enseignants déclarent utiliser un style motivationnel soutenant l'autonomie, et moins les élèves se disent amotivés $(\beta=-0,05)$. De la même manière, plus les élèves perçoivent que leur enseignant soutient leurs besoins, et moins ils se déclarent amotivés $(\beta=-0,14)$. On relève aussi un effet négatif des croyances d'autoefficacité $(\beta=-0,11)$ sur l'amotivation : plus les élèves se sentent capables d'apprendre ce qui est proposé en classe et moins ils se déclarent amotivés.

\section{Expliquer les croyances d'auto-efficacité à partir du style motivationnel de l'enseignant}

En ce qui concerne les croyances d'auto-efficacité, les résultats (modèle D', cf. tableau 5 en annexe) font apparaître que ces dernières ne sont pas reliées directement au style motivationnel autorapporté de l'enseignant. En revanche, on observe une relation significativement positive entre la perception par les élèves du style motivationnel de leur enseignant et leurs croyances d'auto-efficacité : plus les élèves perçoivent que le style motivationnel de leur enseignant soutient leurs besoins et plus ils déclarent se sentir capables d'accomplir les tâches proposées en classe $(\beta=0,22)$.
Le style motivationnel perçu occasionne ici une réduction de $14,6 \%$ de la variance intra-classe.

\section{Expliquer la perception des élèves relative au style motivationnel de leur enseignant à partir du style motivationnel autorapporté par l'enseignant}

Les résultats (modèle E', cf. tableau 6 en annexe) révèlent que, conformément à nos hypothèses, plus les enseignants déclarent soutenir les besoins psychologiques de leurs élèves, plus ces derniers perçoivent ce soutien $(\beta=0,11)$. Cela conduit à une réduction de $9 \%$ de la variance inter-classe (sans affecter la variance intra-classe puisque la variable est mesurée au niveau classe).

\section{Présentation du modèle intégré des acquisitions scolaires}

Nous pouvons maintenant présenter un modèle intégré des acquisitions scolaires (voir figure 3), issu des estimations empiriques des modèles séquentiels testés.

Rappelons que, selon les approches sociocognitives (Bandura, 1986 ; Deci \& Ryan, 2002), les perceptions des élèves médiatisent la relation entre l'environnement social et les processus motivationnels. Pour cette raison, nous avons analysé l'effet du style motivationnel autorapporté par l'enseignant sur la perception des élèves relative au style motivationnel de leur enseignant. Nous avons vu qu'il existe un lien entre ces deux variables, quoique modéré $(\beta=0,11)$. Le style motivationnel autorapporté par l'enseignant est aussi directement relié à l'amotivation, quoique de façon plus faible $(\beta=-0,05)$. En dehors du lien direct avec ces deux variables, le style motivationnel autorapporté par l'enseignant n'est relié qu'indirectement aux processus motivationnels (croyances d'auto-efficacité et motivation autodéterminée), via la perception qu'en ont les élèves. L'impact du style motivationnel autorapporté par l'enseignant sur les acquisitions est donc " distal », puisque transitant via plusieurs processus intermédiaires.

La perception des élèves relative au style motivationnel de leur enseignant a quant à elle une relation directe avec chacune des variables subséquentes du modèle général. Toutes suivent le signe attendu d'après nos postulats théoriques, sauf la relation négative avec les acquisitions scolaires $(\beta=-0,13)$. Plus les élèves disent percevoir des comportements de leur enseignant qui soutiennent leurs besoins et moins leurs scores d'acquisitions sont élevés en fin 


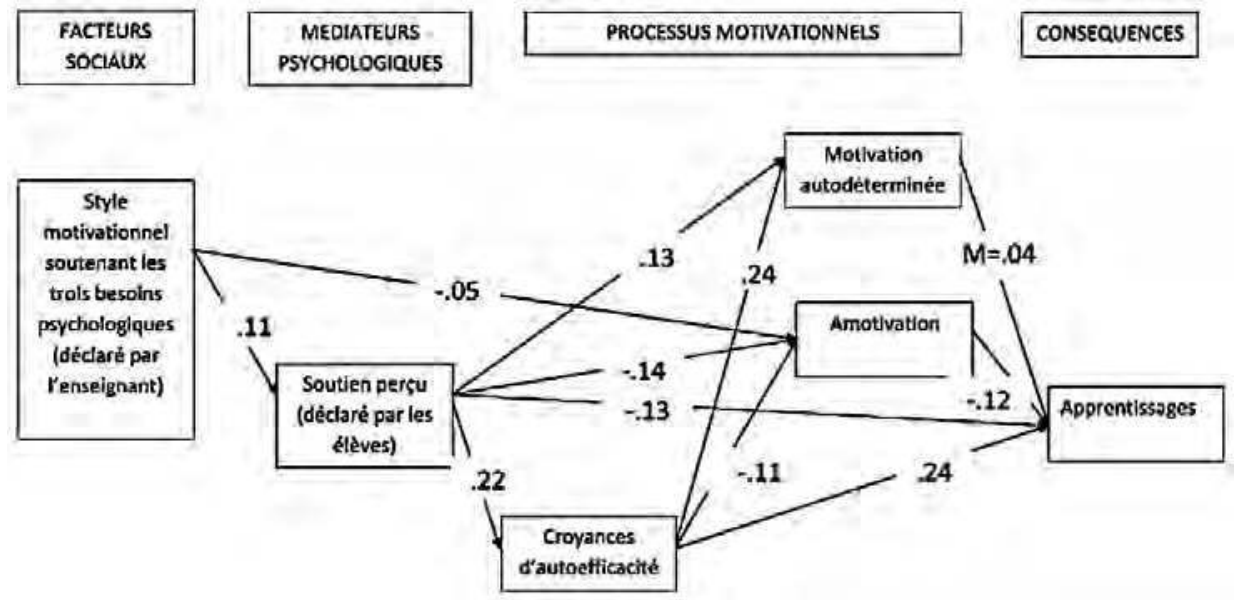

Note : pour la relation entre l'indice d'autodétermination et le score d'acquisitions, on fait figurer la valeur moyenne car la relation est quadratique.

d'année. Par ailleurs, la perception des élèves du style motivationnel de leur enseignant est reliée de manière indirecte aux acquisitions, via l'ensemble des variables motivationnelles qui entretiennent quant à elles une relation directe avec les acquisitions.

Conformément à nos hypothèses, les résultats montrent que si les croyances d'auto-efficacité des élèves sont reliées directement aux apprentissages, ce lien est également indirect dans la mesure où les coefficients directeurs liant cette variable à l'indice d'autodétermination $(\beta=0,24)$ d'une part et au niveau d'amotivation $(\beta=-0,11)$ d'autre part sont eux aussi significatifs. Ainsi, plus les élèves se sentent capables d'accomplir les tâches qui leur sont proposées, plus leur motivation est autodéterminée, une variable qui prédit elle-même de façon globalement positive (quoique non linéaire) leurs acquisitions. En revanche, plus les croyances d'auto-efficacité sont fortes, moins l'amotivation est élevée, variable qui prédit elle-même négativement les acquisitions en fin d'année $(\beta=-0,12)$.

\section{DISCUSSION}

L'objectif de cette étude était de proposer un modèle explicatif des acquisitions scolaires basé sur la théorie de l'autodétermination (voir Deci \& Ryan, 2002) et la théorie sociocognitive de Bandura (1986), modèle selon lequel le style motivationnel de l'enseignant est relié aux acquisitions des élèves, par l'intermédiaire 1) des perceptions que les élèves en ont, et 2) des degrés d'autodétermination, d'amotivation et d'autoefficacité des élèves (voir figure 1). Conformément à la TAD, le modèle prévoit que le style motivationnel d'un enseignant qui soutient les besoins fondamentaux d'autonomie, de compétence et de proximité sociale des élèves est susceptible de nourrir une plus grande motivation autodéterminée et une plus faible amotivation, deux variables motivationnelles qui sont reliées respectivement positivement et négativement aux acquisitions (Reeve, 2002, 2009). Conformément à la théorie sociocognitive de Bandura, les croyances d'auto-efficacité constituent une ressource motivationnelle majeure qui affecte les performances humaines soit directement, soit indirectement par l'intermédiaire de processus motivationnels (Bandura, 1986). Les croyances d'auto-efficacité sont elles-mêmes dépendantes de l'environnement social, en particulier des encouragements et marques de soutien provenant de l'enseignant. Enfin, les deux théories soulignent le rôle médiateur fondamental des "perceptions " de l'environnement social. Autrement dit, ce n'est pas la réalité objective qui compte le plus, mais bien la manière dont l'individu - l'élève en l'occurrence - la 
construit. À cet égard, cette étude est l'une des rares à mesurer le style de l'enseignant à la fois en utilisant les informations de l'enseignant (c'est-à-dire les comportements qu'il dit adopter en classe quand il s'adresse à ses élèves) et celles des élèves (c'est-àdire les perceptions qu'ils ont des comportements de leur enseignant).

Pris dans leur ensemble, les résultats apportent un soutien au modèle présumé et mettent en lumière la complexité des mécanismes impliqués dans la relation enseignement-acquisitions. Nous avons en effet pu constater que, si le style motivationnel autorapporté par l'enseignant est bien relié aux performances scolaires, ce lien est largement médiatisé, d'une part par les perceptions que les élèves ont du style motivationnel de leur enseignant et, d'autre part, par leur motivation (degrés d'autodétermination, d'amotivation et d'auto-efficacité). Plus précisément, les résultats montrent que, conformément à nos hypothèses, le style particulier avec lequel un enseignant interagit au quotidien avec ses élèves est perçu par ces derniers. Quand les élèves ont le sentiment que leur enseignant soutient leur autonomie, leur compétence et leur proximité sociale, ils sont plus à même de manifester une plus grande motivation autodéterminée, une plus faible amotivation et de plus grandes croyances d'auto-efficacité. En retour, une plus grande motivation autodéterminée, une plus faible amotivation et de plus grandes croyances d'auto-efficacité prédisent chacune de meilleures acquisitions.

Un autre résultat intéressant de cette étude concerne le rôle des croyances d'auto-efficacité dans le processus d'internalisation de la motivation. Comme on peut le voir dans la figure 3, l'auto-efficacité est reliée aux acquisitions à la fois directement (par exemple en augmentant les ressources affectives et cognitives engagées par les élèves) et indirectement par les régulations motivationnelles qui ont été mesurées. Plus les croyances d'auto-efficacité sont élevées, plus la motivation de l'élève est autodéterminée et moins il est amotivé, deux variables qui prédisent elles-mêmes les acquisitions comme nous venons de le voir. Ce résultat est conforme à la TAD (Deci \& Ryan, 2000), selon laquelle la satisfaction des besoins psychologiques - en l'occurrence, ici, celui de compétence - facilite l'appropriation des régulations à l'égard d'une tâche. Autrement dit, en donnant des feed-back sur les progrès réalisés et en encourageant les élèves, l'enseignant renforce la confiance qu'ils ont à réaliser les tâches scolaires. Cette plus grande efficacité perçue conduit en retour à des motivations plus autonomes pour les tâches scolaires (par exemple régulation identifiée - l'élève comprend davantage l'intérêt de ce qu'il y a à faire - voire régulation intrinsèque - l'élève prend plus de plaisir à ce qu'il fait). À l'inverse, avec de faibles croyances d'auto-efficacité, l'élève tend à réaliser les tâches scolaires non pas de manière autonome mais pour des raisons plus contrôlées comme la régulation introjectée (parce qu'il se sentirait coupable de ne pas les faire) ou la régulation externe (parce qu'il sent des pressions externes comme les punitions). II tend également à être plus amotivé ou « résigné ", un phénomène mis en évidence par Seligman (1975).

Certains résultats inattendus trouvés dans cette étude méritent d'être soulignés. Tout d'abord, si conformément aux postulats des approches sociocognitives, les analyses ont confirmé le rôle de " filtre » (c'est-à-dire de médiateur) exercé par les perceptions des élèves dans la relation unissant le style motivationnel de l'enseignant aux processus motivationnels des élèves, force est de constater que le lien entre les comportements autorapportés par les enseignants d'une part et les perceptions qu'en ont les élèves d'autre part est faible $(\beta=0,11)$. Autrement dit, la congruence entre les points de vue des enseignants et des élèves en ce qui concerne le style motivationnel de l'enseignant n'est pas très importante. Même s'il existe une tendance à la ressemblance des points de vue, il n'en demeure pas moins que les perceptions des élèves sont loin d'être déterminées entièrement par ce que l'enseignant déclare faire en classe. Une observation similaire a été faite par Skinner et Belmont (1993) dans une étude conduite avec des élèves âgés de 8 à 12 ans, dans laquelle les relations trouvées entre les différentes dimensions du style motivationnel de l'enseignant et les perceptions qu'en avaient les élèves s'étalaient entre 0,03 et 0,28 (en termes de coefficients bêtas). Trois explications principales peuvent être apportées : un manque d'objectivité des enseignants, un biais dans la perception des élèves et un décalage dans le niveau d'analyse. Tout d'abord, il est probable que les déclarations de l'enseignant ne correspondent pas tout à fait à la réalité des comportements qu'il met en œuvre. Même si le questionnaire était anonyme, l'existence de biais de désirabilité sociale n'est pas à exclure. Ces derniers ont pu conduire les enseignants à mentionner davantage ce qu'ils pensaient être bien de dire (les comportements qui soutiennent les besoins des élèves) que ce qu'ils faisaient réellement. La moyenne très élevée des scores autorapportés par les enseignants ( $M=4,32$ sur une échelle de 5 points) conforte cette possibilité. D'autre part, il est probable que les élèves ne perçoivent pas avec une complète exactitude les comportements 
véritables de l'enseignant. L'occurrence de biais perceptifs n'est pas à exclure. II est possible que les élèves qui aiment bien leur enseignant voient chez lui davantage de comportements positifs qu'il n'en manifeste vraiment, et inversement. En l'absence d'indicateurs objectifs (par exemple une observation faite par une tierce personne) des comportements réellement manifestés par l'enseignant, il est difficile de trancher en faveur de l'une ou l'autre de ces deux explications. Une troisième explication possible provient du décalage qui existe dans les niveaux d'analyse. Rappelons que le style motivationnel de l'enseignant est évalué à la fois au niveau de l'enseignant (ce qu'il dit faire en général) et des élèves (ce que chaque élève perçoit de son enseignant). Or, il est possible que les comportements que l'enseignant dit mettre en œuvre "généralement » masquent une variabilité importante quand il s'adresse à " chacun " de ses élèves. Dès lors, la corrélation entre les deux mesures est faible, simplement parce que la mesure au niveau de l'enseignant manque de spécificité. Peut-être que les résultats auraient été sensiblement différents si on lui avait demandé de remplir le questionnaire pour chacun de ses élèves, tâche fastidieuse qui n'était pas envisageable dans le cadre de cette étude.

Un autre résultat inattendu concerne le lien direct qui existe entre le style motivationnel autorapporté par l'enseignant et l'amotivation. Cette relation négative ne semble pas médiatisée par la perception des élèves relative au style motivationnel de leur enseignant. Deux explications peuvent être apportées à ce résultat. Tout d'abord, il est possible que le questionnaire de perceptions par les élèves relatives au style motivationnel de leur enseignant ne soit pas assez précis pour capturer tous les processus susceptibles de conduire à l'amotivation. En effet, le questionnaire tend à mesurer uniquement les comportements " de soutien " des besoins. Or, il est possible que ce soit davantage la présence de comportements qui « menacent » les besoins (par exemple "Mon enseignant me dit que je suis nul »), plus que l'absence de comportements qui les soutiennent (par exemple "Ce que dit mon enseignant augmente la confiance que j'ai en moi »), qui conduisent à l'amotivation. Des travaux réalisés ces dernières années (voir Reeve, 2009 ; Sarrazin, Pelletier, Deci et al., 2011, pour une revue) révèlent en effet l'intérêt de séparer ces deux types de comportements de l'enseignant. L'autre explication que l'on peut apporter à ce résultat est que les effets du style de l'enseignant sur l'amotivation échappent en partie à la conscience des élèves. Certains travaux sur la contagion des motivations entre l'enseignant et les élèves (notamment Radel, Sarrazin, Legrain et al.,
2010) révèlent l'existence de mécanismes inconscients qui interviennent dans la relation pédagogique.

Enfin, le résultat le plus surprenant de cette étude concerne une relation négative trouvée entre la perception des élèves relative au style motivationnel de leur enseignant et les acquisitions des élèves. Nous avons cherché à savoir si le sens de cette relation pouvait changer en fonction des valeurs prises par les perceptions des élèves, mais la fonction quadratique s'est révélée non significative. Plusieurs hypothèses peuvent être avancées pour expliquer ce résultat inattendu. Une première explication concerne les effets paradoxaux des feed-back délivrés par l'enseignant trouvés dans la littérature. Si la théorie sociocognitive de Bandura (1997) a montré que les feed-back positifs et les encouragements influençaient positivement les croyances d'auto-efficacité, d'autres études ont trouvé que les encouragements pouvaient avoir des effets contrastés sur les acquisitions. Par exemple, plusieurs chercheurs (Cimpian, Arce, Markman et al., 2007 ; Kamins \& Dweck, 1999 ; Mueller \& Dweck, 1998) ont montré que le fait de féliciter un enfant « pour son intelligence " pouvait provoquer chez ce dernier une focalisation sur la démonstration de sa supériorité sociale, alors que les compliments en lien avec les efforts fournis favoriseraient une focalisation sur la maîtrise des tâches. Or, face à la difficulté, les élèves orientés vers la démonstration de la supériorité sociale persisteraient moins que ceux orientés vers la maîtrise, expliquant ainsi les moins bonnes performances des premiers. En mettant en œuvre un ensemble de processus cognitifs, affectifs et comportementaux contrastés, ces focalisations divergentes permettent de mieux comprendre les liens complexes qui unissent la perception des élèves relative au soutien de leur enseignant et les acquisitions scolaires. Sans une mesure spécifique des orientations motivationnelles des élèves, cette interprétation reste néanmoins de l'ordre de l'hypothèse.

Une autre explication possible concerne la relation réciproque qui peut exister entre les perceptions des élèves relatives au climat de leur enseignant et leur niveau de performance. Rappelons qu'il s'agit d'une étude transversale dans laquelle ces deux variables ont été mesurées au même moment. Si notre modèle présume un lien qui irait de la première variable vers la seconde, rien ne permet d'exclure la possibilité d'une relation inverse. II est en effet tout à fait envisageable que les élèves dont les résultats académiques sont les plus faibles bénéficient d'une plus grande attention et d'un plus grand soutien de la part de leur enseignant. À l'inverse, les élèves les plus en réussite à 
l'école peuvent avoir le sentiment que leur enseignant s'occupe un peu moins d'eux. Seule une étude longitudinale qui prendrait en compte les perceptions et les résultats des élèves en début et en fin d'année permettrait d'apporter un soutien plus fort à cette explication.

Ainsi, le caractère synchronique de notre étude en constitue une claire limite. Ce caractère ne permet guère en effet de parler de liens de "causalité " entre les variables. Comme nous l'avons évoqué, des relations réciproques sont tout à fait possibles, de même que des covariations non causales liées à des variables non mesurées. Des études longitudinales avec plusieurs temps de mesure seraient à privilégier à l'avenir pour affirmer avec plus de force les relations de causalité entre les variables. Cela permettrait en effet de préciser le rôle des variables motivationnelles, en particulier du style motivationnel de l'enseignant, dans l'évolution des acquisitions des élèves. Notre analyse a porté sur la seule mesure d'acquisitions disponible dans la base de données EBE de la DEPP, à savoir le score d'acquisitions des élèves en fin d'année scolaire. Nous devons donc insister sur le fait qu'il ne s'agit pas en l'occurrence d'un résultat en termes de progression scolaire puisque le niveau scolaire de départ n'a pu faire l'objet d'une évaluation en début d'année. Disposer d'une variable qui caractérise le niveau d'acquisitions des élèves à l'entrée en CM2 aurait permis de contrôler les différences interindividuelles initiales et de mieux estimer le poids des processus motivationnels générés au cours de la classe de CM2.

Nadia Leroy Nadia.Leroy@unige.ch Université de Genève, groupe Méthodologie et Analyse de Données

Pascal Bressoux Pascal.Bressoux@upmf-grenoble.fr Université Grenoble-Alpes, laboratoire des Sciences de l'Éducation et Institut universitaire de France

Philippe Sarrazin philippe.sarrazin@ujf-grenoble.fr Université Grenoble-Alpes, laboratoire Sport et ENvironnement Social

David Trouilloud david.trouilloud@ujf-grenoble.fr Université Grenoble-Alpes, laboratoire Sport et ENvironnement Social

\section{NOTE}

1 La spécification des $M M N$ a été effectuée étape par étape avec introduction successive de variables explicatives. Toutefois, par souci de place, nous ne présenterons pas les modèles intermédiaires. Nous ne présentons que les estimations qui répondent de manière satisfaisante aux critères d'ajustement (effets fixes significatifs, réduction significative de la part de variance résiduelle et décroissance significative de la déviance $\Delta_{D}$ ).

\section{BIBLIOGRAPHIE}

ANDERSON R. C. (1970). " Control of student mediating processes during verbal learning and instruction ". Review of Educational Research, no 40(3), p. 349-369.

BANDURA A. (1986). Social foundations of thought and action. A social cognitive theory. Englewood Cliffs (États-Unis) : Prentice-Hall.

BANDURA A. (1997). Self-efficacy: The exercise of control. New York : Freeman.

BAUMEISTER R. F. \& LEARY M. R. (1995). « The need to belong: Desire for interpersonal attachments as a fundamental human motivation ». Psychological Bulletin, no 117, p. 497-529.

BLACK A. E. \& DECI E. L. (2000). "The effects of instructors' autonomy support and students' autonomous motivation on learning organic chemistry: A self-determination theory perspective ". Science Education, no 84, p. 740-756.

BOGGIANO A. K. (1998). « Maladaptive achievement patterns: A test of a diathesis-stress analysis of helplessness ". Journal of Personality and Social Psychology, no 74, p. 1681-1695.

BRESSOUX P. (1994). "Les recherches sur les effetsécoles et les effets-maîtres ». Revue française de pédagogie, no 108, p. 91-137.

BRESSOUX P. (2010). Modélisation statistique appliquée aux sciences sociales. Bruxelles: De Boeck (2e éd.).

CHIRKOV V. I. \& RYAN R. M. (2001). "Parent and teacher autonomy-support in Russian and U.S. adolescents: Common effects on well-being and academic motivation ". Journal of Cross-Cultural Psychology, n० 32(5), p. 618-635.

CIMPIAN A., ARCE H., MARKMAN E. M. \& DWECK C. S. (2007). "Subtle linguistic cues impact children's motivation ». Psychological Science, no 18, p. 314316.

De CHARMS R. (1968). Personal causation: The internal affective determinants of behavior. New York : Academic press. 
DECI E. L. (1975). Intrinsic motivation. New York : Plenum Press.

DECI E. L., CONNELL J. P. \& RYAN R. M. (1989). "Selfdetermination in a work organization ". Journal of Applied Psychology, n० 74, p. 580-590.

DECI E. L., KOESTNER R. \& RYAN R. M. (1999). «A meta-analytic review of experiments examining the effects of extrinsic rewards on intrinsic motivation ". Psychological Bulletin, no 125, p. 627-668.

DECI E. L. \& RYAN R. M. (1987). "The support of autonomy and the control of behavior ". Journal of Personality and Social Psychology, no 53, p. 1024-1037.

DECI E. L. \& RYAN R. M. (2000). "The "what" and "why" of goal pursuits: human needs and the Self-Determination of behaviour ». Psychology Inquiry, no 11, p. 227-268.

DECI E. L. \& RYAN R. M. (2002). Handbook of Selfdetermination research. Rochester (États-Unis) : The University of Rochester Press.

DOYLE W. (1986). «Paradigmes de recherche sur l'efficacité des enseignants ». In M. Crahay \& D. Lafontaine (coord.), L'art et la science de l'enseignement. Bruxelles : Éd. Labor, p. 435-481.

GOETZ T., CRONJAEGER H., FRENZEL A. C., LÜDTKE O. \& HALL N. C. (2010). "Academic self concept and emotion relations: Domain specificity and age effects ". Contemporary Educational Psychology, n० 35 , p. 44-58.

GOOD T. L. \& BROPHY J. E. (2000). Looking in classrooms. New York : Longman (8e éd.).

GROLNICK W. S. \& SLOWIACZEK M. (1994). "Parents' involvement in children's schooling: A multidimensional conceptualization and motivational model ". Child Development, n॰65, p. 237-252.

KAMINS M. \& DWECK C. S. (1999). «Person vs. process praise and criticism: Implications for contingent selfworth and coping ". Developmental Psychology, n॰ 35, p. 835-847.

KAPLAN A. \& MAEHR M. L. (1999). "Enhancing the motivation of African American students: An achievement goal theory perspective ". Journal of Negro Education, n०68(1), p. 23-41.

LEVIE W. H. \& DICKIE K. (1973). "The analysis and application of media ". In R. Travers (dir.), The second handbook of research on teaching. Chicago : Rand McNally.

MUELLER C. M. \& DWECK C. S. (1998). " Intelligence praise can undermine motivation and performance ". Journal of Personality and Social Psychology, n० 75, p. 33-52.

NIEMIEC C. P. \& RYAN R. M. (2009). " Autonomy, competence, and relatedness in the classroom: Applying self-determination theory to educational practice ". Theory and Research in Education, n० 7 , p. 133-144.

PAJARÈS F. (1996). "Self-efficacy beliefs in achievement settings ". Review of Educational Research, n० 66, p. 543-578.

PIETSCH J., WALKER R. \& CHAPMAN E. (2003). "The relationship among self-concept, self-efficacy, and performance in mathematics during secondary school ». Journal of Educational Psychology, no 95(3), p. 589-603.

PINTRICH P. R. \& De GROOT E. V. (1990). « Motivational and self-regulated learning components of classroom academic performance ". Journal of Educational Psychology, no 82, p. 33-40.

PINTRICH P. R. \& SCHUNK D. H. (1996). Motivation in Education: Theory, Research \& Applications. Englewood Cliffs (États-Unis) : Prentice-Hall.

RADEL R., SARRAZIN P., LEGRAIN P. \& WILD C. (2010). "Social Contagion of Motivation Between Teacher and Student: Analyzing Underlying Processes ». Journal of Educational Psychology, no 102, p. 577-587.

REEVE J. (2002). "Self-determination theory applied to educational setting ». In E. L. Deci \& R. M. Ryan (dir.), Handbook of self-determination research. Rochester (États-Unis) : University of Rochester Press, p. 183203.

REEVE J. \& JANG H. (2006). «What teachers say and do to support students' autonomy during a learning activity ". Journal of Educational Psychology, n० 98, p. 209-218.

REEVE J. (2009). "Why teachers adopt a controlling motivating style toward students and how they can become more autonomy supportive ". Educational Psychologist, no 44(3), p. 159-178.

RYAN R. M. \& CONNELL J. P. (1989). «Perceived locus of causality and internalization: Examining reasons for acting in two domains ". Journal of Personality and Social Psychology, n० 57, p. 749-761.

RYAN R. M. \& DECI E. L. (2000). "Intrinsic and extrinsic motivations: Classic definitions and new directions". Contemporary Educational Psychology, n० 25, p. 5467.

RYAN R. M. \& GROLNICK W. S. (1986). "Origins and Pawns in the Classroom: Self-Report and Projective Assessment of Individual Differences in Children's Perceptions ". Journal of Personality and Social Psychology, no 45, p. 736-750.

RYAN R. M. \& PATRICK H. (2001). "The classroom social environment and changes in adolescents' motivation and engagement during middle school ". American Educational Research Journal, n 38, p. 437-460.

SARRAZIN P., PELLETIER L., DECI E. L. \& RYAN R. M (2011). "Nourrir une motivation autonome et des conséquences positives dans différents milieux de vie : les apports de la théorie de l'autodétermination ". In C. Martin-Krumm \& C. Tarquinio (dir.), Traité de psychologie positive. Bruxelles: De Boeck, p. 273312.

SCHUNK D. H. (1991). "Self-efficacy and academic motivation ». Educational Psychologist, n०26, p. 207232.

SELIGMAN M.E.P. (1975). Helplessness: On Depression, Development, and Death. San Francisco: W. H. Freeman.

SHUELL T. J. (1996). " Teaching and learning in a classroom context ". In D. C. Berliner \& R. C. Calfee (dir.), Handbook of educational psychology. New York : Macmillan, p. 726-764. 
SKAALVIK E. M. \& HAGTVET K. (1990). « Academic achievement and self-concept: An analysis of causal predominance in a developmental perspective ". Journal of Personality and Social Psychology, no 58, p. 292-307.

SKINNER E. A., \& BELMONT M. J. (1993). "Motivation in the classroom: Reciprocal effects of teacher behavior and student engagement across the school year ". Journal of Educational Psychology, n॰ 85(5), p. 71-581.

TESSIER D., SARRAZIN P. \& TROUILLOUD D. (2006). " Climat motivationnel instauré par l'enseignant et implication des élèves en classe : l'état des recherches ". Revue française de pédagogie, n 157, p. 147-177.

VALLERAND R. J \& BISSONNETTE R. (1992). «Intrinsic, extrinsic, and amotivational styles as predictors of behavior: A prospective study ». Journal of Personality, n० 60, p. 599-620.
WHITE R. W. (1959). " Motivation reconsidered. The concept of competence ». Psychological Review, no 66, p. 297-333.

WIGFIELD A., ECCLES J. S., YOON K. S., HAROLD R.D., ARBRETON A., FREEDMAN-DOAN C. et al. (1997). « Changes in children's competence beliefs andsubjective task values across the elementary school years: A three-year study ». Journal of Educational Psychology, no 89, p. 451-469.

WILLIAMS G. C. \& DECI E. L. (1996). « Internalization of biopsychosocial values by medical students: A test of self-determination theory ". Journal of Personality and Social Psychology, no 70, p. 767-779.

WILLIAMS G. C., FREEDMAN Z. R. \& DECI E. L. (1998). «Supporting autonomy to motivate glucose control in patients with diabetes ". Diabetes Care, no 21, p. 1644-1651.

\section{ANNEXE}

Lecture des tableaux où figurent les estimations multi-niveaux : les effets fixes représentent l'impact des variables indépendantes sur la variable dépendante. Les coefficients sont assortis de leur erreur-type (entre parenthèses) et de leur significativité.

Lorsque la variable indépendante est quantitative, le coefficient traduit l'impact d'une unité de variation de cette variable sur la variable dépendante. Par exemple, dans le tableau 2 (modèle A'), une augmentation d'une unité des croyances d'auto-efficacité personnelle augmente de 15,33 points le score d'acquisitions des élèves.

Lorsque la variable indépendante est qualitative, le coefficient traduit l'écart sur la variable dépendante entre une modalité de la variable indépendante et une autre considérée comme modalité de référence. Par exemple, dans le tableau 2 (modèle $A$ bis), les enfants dont le père exerce une profession intermédiaire obtiennent un score d'acquisitions qui est en moyenne inférieur de 11,73 points à celui des enfants de cadres supérieurs et professions intellectuelles supérieures (modalité de référence).

Les effets aléatoires représentent la répartition de la variance de la variable dépendante selon les deux niveaux de la hiérarchie : les élèves (variance intra-classe) et les classes (variance inter-classes). 
Tableau 2. Modèles multi-niveaux estimant les scores totaux d'acquisitions des élèves

\begin{tabular}{|c|c|c|c|}
\hline Variables & Modèle A (vide) & Modèle A bis & Modèle A' \\
\hline \multicolumn{4}{|l|}{ Effets fixes } \\
\hline Constante & $254,43(1,32)^{\star \star \star}$ & $250,41(4,51)^{\star \star \star}$ & $257,79(9,04)^{\star \star \star}$ \\
\hline \multicolumn{4}{|l|}{$\begin{array}{l}\text { Variables contrôlées } \\
\text { Profession du père (référence }\end{array}$} \\
\hline Profession intermédiaire & & $-11,73(2,25)^{\star \star \star}$ & $-9,05(2,11)^{\star * *}$ \\
\hline Employé & & $-17,71(2,57)^{\star \star \star}$ & $-13,80(2,41)^{\star \star \star}$ \\
\hline Artisan & & $-17,71(2,51)^{\star \star \star}$ & $-14,14(2,36)^{\star \star \star}$ \\
\hline Agriculteur & & $-16,16(4,03)^{\star \star *}$ & $-13,18(3,78)^{\star \star}$ \\
\hline Ouvrier & & $-30,25(2,21)^{\star \star *}$ & $-24,70(2,09)^{\star \star *}$ \\
\hline Autre & & $-20,49(2,40)^{* \star *}$ & $-16,23(2,27)^{\star * *}$ \\
\hline \multicolumn{4}{|l|}{ Fratrie (référence aucun frère ou sœur) } \\
\hline Un frère ou sœur & & $-2,33(2,41) n s$ & $-0,28(2,26) n s$ \\
\hline Deux frères ou sœurs & & $-1,41(2,48) n s$ & $\begin{array}{l}1,22(2,33) \mathrm{ns} \\
-417(2,65) \mathrm{ns}\end{array}$ \\
\hline Trois frères ou sœurs & & $-6,47(2,84)^{\star}$ & $\begin{array}{l}-4,17(2,65) n s \\
-6,47(279)^{\star *}\end{array}$ \\
\hline Plus de trois frères ou sœurs & & $-11,46(2,98)^{\star \star \star}$ & \\
\hline \multicolumn{4}{|l|}{ Période naissance (référence début d'année) } \\
\hline Milieu d'année & & $-1,37(1,49) n s$ & \\
\hline Fin d’année & & $-2,72(1,52) n s$ & $-2,31(1,42)$ ns \\
\hline Non renseigné & & $-19,93(6,26)^{\star \star \star}$ & \\
\hline \multicolumn{4}{|l|}{ Déroulement scolarité (référence retard) } \\
\hline Heure & & $35,79(3,31)^{\star \star \star}$ & $29,89(3,12)^{\star \star \star}$ \\
\hline Avance & & $55,20(4,66)^{\star \star \star}$ & $45,82(4,39)^{\star \star \star}$ \\
\hline \multicolumn{4}{|r|}{$-19,90(1,88)^{\star \star *}$} \\
\hline Redoublement & & $10,17(3,61)^{\star}$ & $-9,98(3,39)^{\star \star \star}$ \\
\hline \multicolumn{4}{|l|}{ Indice d'autodétermination } \\
\hline Indice d'autodétermination quadratique & & & $\begin{array}{c}0,31(0,05)^{\star \star *} \\
{[\beta=0,04]}\end{array}$ \\
\hline Amotivation & & & $\begin{array}{c}-6,80(0,78)^{\star * *} \\
{[\beta=-0,12]^{1}}\end{array}$ \\
\hline Croyances d'auto-efficacité & & & $\begin{array}{c}15,33(0,83)^{\star * *} \\
{[\beta=0,24]}\end{array}$ \\
\hline Style motivationnel de l'enseignant perçu par les élèves & & & $\begin{array}{c}-8,02(0,76)^{\star \star \star} \\
{[\beta=-0,13]}\end{array}$ \\
\hline Style motivationnel autorapporté par l'enseignant & & & ns \\
\hline \multicolumn{4}{|l|}{ Effets aléatoires } \\
\hline Part de variance inter-classes & $\begin{array}{c}378,94 \\
(43,89)^{\star * \star}\end{array}$ & $219,75(28,10)^{\star \star \star}$ & $\begin{array}{c}234,74 \\
(28,64)^{\star \star \star}\end{array}$ \\
\hline Part de variance intra-classe & $\begin{array}{l}1995,31 \\
(46,5)^{\star \star \star}\end{array}$ & $\begin{array}{c}1565,43 \\
(34,63)^{\star \star \star}\end{array}$ & $\begin{array}{c}1357,57 \\
(30,16)^{\star \star \star}\end{array}$ \\
\hline$-2 \log L$ & 46330,90 & 45199,90 & 44403,30 \\
\hline$\Delta_{D}$ & & 1131 & $1927,60 * * *$ \\
\hline
\end{tabular}

Notes : 1 : pour des raisons de commodité de lecture des coefficients quadratiques, l'échelle de l'indice d'autodétermination a fait l'objet d'une translation de telle manière que toutes ses valeurs soient positives $(M=11,12 ; E T=2,88 ; \min =0,54 ; \max =21,67)$. On donne ici l'estimation de $\beta$ pour une valeur moyenne de l'indice d'autodétermination; $N=4402 ;{ }^{*}: p<0,05 ;{ }^{\star \star}: p<0,001 ;{ }^{\star \star \star}: p<0,0001$. 
Tableau 3. Modèles multi-niveaux estimant l'indice d'autodétermination des élèves

\begin{tabular}{|c|c|c|}
\hline Variables & Modèle B (vide) & Modèle B' \\
\hline \multicolumn{3}{|l|}{ Effets fixes } \\
\hline Constante & $11,13(0,06)^{\star \star \star}$ & $5,57(0,67)^{\star \star \star}$ \\
\hline \multicolumn{3}{|l|}{ Variables contrôlées } \\
\hline Profession du père (référence cadre sup.) & & \\
\hline Profession intermédiaire & & ns \\
\hline Employé & & ns \\
\hline Artisan & & ns \\
\hline Agriculteur & & ns \\
\hline Ouvrier & & ns \\
\hline Autre & & ns \\
\hline \multicolumn{3}{|l|}{ Fratrie (référence aucun frère ou sœur) } \\
\hline Un frère ou sœur & & ns \\
\hline Deux frères ou sœurs & & ns \\
\hline Trois frères ou sœurs & & ns \\
\hline Plus de trois frères ou sœurs & & ns \\
\hline \multicolumn{3}{|l|}{ Période naissance (référence début d'année) } \\
\hline Milieu d'année & & ns \\
\hline Fin d'année & & ns \\
\hline Non renseigné & & ns \\
\hline \multicolumn{3}{|l|}{ Déroulement scolarité (référence retard) } \\
\hline Heure & & $0,29(0,12)^{\star \star}$ \\
\hline Avance & & $0,52(0,24)^{*}$ \\
\hline Soutien RASED & & $-0,43(0,13)^{\star *}$ \\
\hline Redoublement & & ns \\
\hline Croyances d'auto-efficacité & & $\begin{array}{c}0,90(0,07)^{\star \star \star} \\
{[\beta=0,24]}\end{array}$ \\
\hline Style motivationnel de l'enseignant perçu par les élèves & & $\begin{array}{c}0,45(0,05)^{\star \star \star} \\
{[\beta=0,13]}\end{array}$ \\
\hline Style motivationnel autorapporté par l'enseignant & & $\mathrm{ns}$ \\
\hline \multicolumn{3}{|l|}{ Effets aléatoires } \\
\hline Part de variance inter-classes & $0,49(0,08)^{\star \star \star}$ & $0,40(0,07)^{\star \star \star}$ \\
\hline Part de variance intra-classe & $7,80(0,17)^{\star \star \star}$ & $7,01(0,15)^{\star \star \star}$ \\
\hline$-2 \log L$ & 21723,20 & 21249,80 \\
\hline$\Delta_{\mathrm{D}}$ & & $473,40^{\star \star \star}$ \\
\hline
\end{tabular}

Note $: \mathrm{N}=4402 ;{ }^{*}: \mathrm{p}<0,05 ;{ }^{\star \star}: \mathrm{p}<0,001 ;{ }^{* \star \star}: \mathrm{p}<0,0001$. 
Tableau 4. Modèles multi-niveaux estimant l'amotivation des élèves

\begin{tabular}{|c|c|c|}
\hline Variables & Modèle C (vide) & Modèle C' \\
\hline \multicolumn{3}{|l|}{ Effets fixes } \\
\hline Constante & $1,59(0,02)^{\star \star \star}$ & $3,16(0,19)^{\star \star \star}$ \\
\hline \multicolumn{3}{|l|}{ Variables contrôlées } \\
\hline Profession du père (référence cadre sup.) & & \\
\hline Profession intermédiaire & & ns \\
\hline Employé & & ns \\
\hline Artisan & & ns \\
\hline Agriculteur & & ns \\
\hline Ouvrier & & ns \\
\hline Autre & & ns \\
\hline \multicolumn{3}{|l|}{ Fratrie (référence aucun frère ou sœur) } \\
\hline Un frère ou sœur & & ns \\
\hline Deux frères ou sœurs & & ns \\
\hline Trois frères ou sœurs & & ns \\
\hline Plus de trois frères ou sœurs & & ns \\
\hline \multicolumn{3}{|l|}{ Période naissance (référence début d'année) } \\
\hline Milieu d'année & & ns \\
\hline Fin d'année & & ns \\
\hline Non renseigné & & ns \\
\hline \multicolumn{3}{|l|}{ Déroulement scolarité (référence retard) } \\
\hline Heure & & $-0,19(0,03)^{\star \star}$ \\
\hline Avance & & $-0,31(0,07)^{\star}$ \\
\hline Soutien RASED & & $0,17(0,04)^{\star \star}$ \\
\hline Redoublement & & ns \\
\hline \multicolumn{2}{|l|}{ Croyances d'auto-efficacité } & $\begin{array}{c}-0,13(0,02)^{\star \star \star} \\
{[\beta=-0,11]}\end{array}$ \\
\hline \multicolumn{2}{|l|}{ Style motivationnel de l'enseignant perçu par les élèves } & $\begin{array}{c}-0,14(0,01)^{\star * *} \\
{[\beta=-0,14]}\end{array}$ \\
\hline \multicolumn{2}{|l|}{ Style motivationnel autorapporté par l'enseignant } & $\begin{array}{c}-0,11(0,04)^{\star \star} \\
{[\beta=-0,05]}\end{array}$ \\
\hline \multicolumn{3}{|l|}{ Effets aléatoires } \\
\hline Part de variance inter-classes & $0,04(0,007)^{\star \star \star}$ & $0,03(0,006)^{\star \star \star}$ \\
\hline Part de variance intra-classe & $0,69(0,01)^{\star \star \star}$ & $0,65(0,01)^{\star \star \star}$ \\
\hline$-2 \log L$ & 11032,30 & 10723,60 \\
\hline$\Delta_{\mathrm{D}}$ & & $308,70^{\star * *}$ \\
\hline
\end{tabular}

Note $: N=4402 ;{ }^{*}: p<0,05 ;{ }^{* *}: p<0,001 ;{ }^{* * *}: p<0,0001$. 
Tableau 5. Modèles multi-niveaux estimant les croyances d'auto-efficacité des élèves

\begin{tabular}{|c|c|c|}
\hline Variables & Modèle D (vide) & Modèle D' \\
\hline \multicolumn{3}{|l|}{ Effets fixes } \\
\hline Constante & $3,61(0,01)^{\star \star \star}$ & $3,11(0,07)^{\star \star \star}$ \\
\hline \multirow{2}{*}{\multicolumn{3}{|c|}{ Variables contrôlées }} \\
\hline & & \\
\hline Profession intermédiaire & & $-0,13(0,039)^{\star \star \star}$ \\
\hline Employé & & $-0,19(0,044)^{\star \star \star}$ \\
\hline Artisan & & $-0,20(0,043)^{\star * *}$ \\
\hline Agriculteur & & $-0,240(0,07)^{\star \star \star}$ \\
\hline Ouvrier & & $-0,258(0,03)^{\star \star \star}$ \\
\hline Autre & & $-0,21(0,04)^{\star \star \star}$ \\
\hline \multicolumn{3}{|l|}{ Fratrie (référence aucun frère ou sœur) } \\
\hline Un frère ou sœur & & $-0,09(0,04)^{\star}$ \\
\hline Deux frères ou sœurs & & $-0,09(0,04)^{\star}$ \\
\hline Trois frères ou sœurs & & $-0,11(0,05)^{\star}$ \\
\hline Plus de trois frères ou sœurs & & $-0,20(0,05)^{\star * *}$ \\
\hline \multicolumn{3}{|l|}{ Période naissance (référence début d'année) } \\
\hline Milieu d'année & & $-0,03(0,02) n s$ \\
\hline Fin d’année & & $-0,06(0,03)^{\star}$ \\
\hline Non renseigné & & $-0,23(0,11)^{\star}$ \\
\hline \multicolumn{3}{|l|}{ Déroulement scolarité (référence retard) } \\
\hline Heure & & $0,21(0,03)^{\star \star \star}$ \\
\hline Avance & & $0,40(0,07)^{\star \star \star}$ \\
\hline Soutien RASED & & $-0,28(0,03)^{\star \star \star}$ \\
\hline Redoublement & & ns \\
\hline Style motivationnel de l'enseignant perçu par les élèves & & $\begin{array}{c}0,20(0,01)^{\star \star \star} \\
{[\beta=0,22]}\end{array}$ \\
\hline Style motivationnel autorapporté par l'enseignant & & ns \\
\hline \multicolumn{3}{|l|}{ Effets aléatoires } \\
\hline Part de variance inter-classes & $0,01(0,005)^{\star \star \star}$ & $0,02(0,005)^{\star \star \star}$ \\
\hline Part de variance intra-classe & $0,56(0,01)^{\star \star \star}$ & $0,49(0,01)^{\star \star \star}$ \\
\hline$-2 \log L$ & 10054,40 & 9492,60 \\
\hline$\Delta_{\mathrm{D}}$ & & $591,80^{\star \star \star *}$ \\
\hline
\end{tabular}

Note $: \mathrm{N}=4402 ;{ }^{*}: \mathrm{p}<0,05 ;{ }^{* \star}: \mathrm{p}<0,00 ;{ }^{\star \star *}: \mathrm{p}<0,0001$. 
Tableau 6. Modèles multi-niveaux estimant le soutien perçu par les élèves

\begin{tabular}{|c|c|c|}
\hline Variables & Modèle E (vide) & Modèle E' \\
\hline \multicolumn{3}{|l|}{ Effets fixes } \\
\hline Constante & $3,25(0,02)^{\star \star \star}$ & $2,18(0,24)^{\star \star \star}$ \\
\hline $\begin{array}{l}\text { Variables contrôlées } \\
\text { Profession du père (référence cadre sup.) } \\
\text { Profession intermédiaire } \\
\text { Employé } \\
\text { Artisan } \\
\text { Agriculteur } \\
\text { Ouvrier } \\
\text { Autre }\end{array}$ & & $\begin{array}{c}0,01(0,04)^{\star} \\
0,04(0,05) \mathrm{ns} \\
0,08(0,05)^{\star} \\
0,01(0,07) \mathrm{ns} \\
0,12(0,04)^{\star \star} \\
0,08(0,04)^{\star}\end{array}$ \\
\hline $\begin{array}{l}\text { Fratrie (référence aucun frère ou sœur) } \\
\text { Un frère ou sœur } \\
\text { Deux frères ou sœurs } \\
\text { Trois frères ou sœurs } \\
\text { Plus de trois frères ou sœurs }\end{array}$ & & $\begin{array}{l}\text { ns } \\
\text { ns } \\
\text { ns } \\
\text { ns }\end{array}$ \\
\hline $\begin{array}{l}\text { Période naissance (référence début d'année) } \\
\text { Milieu d'année } \\
\text { Fin d'année } \\
\text { Non renseigné }\end{array}$ & & $\begin{array}{l}\text { ns } \\
\text { ns } \\
\text { ns }\end{array}$ \\
\hline $\begin{array}{l}\text { Déroulement scolarité (référence retard) } \\
\text { Heure } \\
\text { Avance }\end{array}$ & & $\begin{array}{l}\text { ns } \\
\text { ns }\end{array}$ \\
\hline $\begin{array}{l}\text { Soutien RASED } \\
\text { Redoublement }\end{array}$ & & $\begin{array}{l}\text { ns } \\
\text { ns }\end{array}$ \\
\hline Style motivationnel autorapporté par l'enseignant & & $\begin{array}{c}0,23(0,05)^{\star * \star} \\
{[\beta=0,11]}\end{array}$ \\
\hline \multicolumn{3}{|l|}{ Effets aléatoires } \\
\hline Part de variance inter-classes & $0,11(0,01)^{\star \star *}$ & $0,10(0,01)^{\star * *}$ \\
\hline Part de variance intra-classe & $0,57(0,01)^{\star \star \star}$ & $0,57(0,01)^{\star \star \star}$ \\
\hline$-2 \log L$ & 10446,50 & 10418,40 \\
\hline$\Delta_{\mathrm{D}}$ & & $28,10^{\star \star \star}$ \\
\hline
\end{tabular}

Note $: N=4402 ;{ }^{*}: p<0,05 ;{ }^{* *}: p<0,001 ;{ }^{* \star *}: p<0,0001$. 